\title{
LETTERS
}

\section{Single electronic medical record for Canada: a second opinion}

Dr. Nav Persaud's editorial published in CMAJ ${ }^{1}$ describes the author's desire for and the need in Canada for a single national electronic medical record (EMR) in primary care. Dr. Persaud speculates that many of our problems in primary care could be solved by this, from sharing of records between clinics, to research, to specialist access to notes. He cites single health systems in the United States and Singapore as examples of how and why this could work. This is an interesting perspective, but one that may be overly simplistic and does not shine light on the whole picture.

Substantial progress and development have been made in the digital health space in Ontario over the past 10 years, and this should be recognized and celebrated. These advances, coupled with a relentless focus on systems integration across the continuum of care, is where we should put our energy.

Although we have much in common, as family doctors across the country, we all ask very different things from our EMRs. Practice environments are not the same in inner-city urban centres, suburban practices, rural areas, university health clinics or care centres for Indigenous people. This complexity of delivery must be appreciated. Nationally, more than $80 \%$ of family doctors have already purchased an EMR system that works for them and are using it fulsomely. In Ontario, this number is more than $85 \%$.

Physicians own their systems. They have made substantial investments in these: financially; in time spent recording information about their patients; and in blood, sweat and tears, producing clinically useful data. We should appreciate this effort and use the systems to their maximum. Much has been accomplished, and advancing from where we are is a very tenable option.

Switching to a single EMR solution that attempts to meet the needs of 43500 family doctors nationally is an impossible task. And it would take decades to recover from the disruption in care created by "ripping and replacing." Canadian provinces have collectively invested billions of dollars in EMR development, deployment and mature use. We cannot afford, as a society or at any level of government, to start over again. That said, fundamental reform of the health system, advances in integrated care models and associated reform of compensation should be the drivers that define how integrated digital health platforms can and should be presented the local, regional, provincial and national level.

Provincial surveys show that satisfaction ratings with individual EMRs are actually quite high. ${ }^{2}$ Even with multiple EMRs on the market, connectivity is improving year over year (witness Netcare in Alberta, ConnectingOntario and ClinicalConnect in Ontario, and Saskatchewan's eHealth portal). In Ontario, information from virtually every hospital is pushed directly into EMRs within minutes of it being generated via Health Report Manager. Laboratory results from everywhere can be queried and downloaded through the Ontario Laboratories Information System. Integration with drug and immunization repositories is occurring now. eReferral and eConsult systems are up and running and are becoming more and more integrated into the point of care.

As we evolve into an increasingly cloud-based environment, the perceived advantages of a single EMR product or database are no longer compelling. What is most helpful is gaining access to data for clinical, research and system planning purposes. This is less a technical issue than a policy one. Integration and interconnectivity are key. We are getting closer and closer to this daily, with the advent of single sign-on, contextual launching of external digital tools from the EMR, data standards and data movement.

The most unpredictable factor is a human one: even when standards exist, having people use them consistently is a challenge. A single EMR does not fix this. Endless dropdown lists and tick boxes do not, either. In primary care, nothing is more important than the patient narrative. We can never lose this.

Choosing a single EMR vendor has other dangers, in terms of creating a monopolistic environment. This makes us vulnerable as a health system and as a profession. Currently, Ontario has certified 11 EMR vendors and 12 EMR products to a set of core requirements that improve constantly over time. This allows progress to occur incrementally on interconnectivity, data portability and system functionality. Having a single vendor control the entire market means that we are at the whim of one company, whose business interests may not be aligned with those of clinicians or patients. We lose our collective influence.

Open-source software is not the solution to this problem, either. Although it has the advantage of being inexpensive and in some cases free - it has the disadvantage of needing as much, if not more, support as proprietary EMRs do. Most physicians lack the knowledge, skill and desire to program and produce changes in their EMRs themselves. They just want to get down to the work of looking after patients. Multiple different, customized instances of an open-source EMR do not improve the situation over the current state in any way.

On the surface, it may seem that having a selection of EMRs nationally is folly. But experience has shown that competition drives change and innovation. We do not disagree that there are aggravations in navigating from an EMR to a viewer or external portals, but this is a solvable problem. The key is to build bridges allowing access to the data that a clinician requires at the point of care. We maintain privacy, confidentiality and security more effectively this way. Data for secondary use can be liberated easily through these structures, if we create the right policy and business drivers. All of this comes at far less risk and with a far better user experience for the average doctor. Banks have done it. Retailers have done it. We are doing it. 
At OntarioMD, we believe in more choice, not less. We want to encourage new software products to enter the market to speed up the pace of innovation. Some of these are EMRs; some are apps that make an EMR fly. We want to let doctors be doctors, not computer engineers or data scientists. And, ultimately, we think that patients should be the first and last point of approval regarding secondary use of their personal health data. These are our principles. We strongly believe that the best way to accomplish them is via our current approach to EMR selection, certification and improvement. One EMR system for all is simply not a realistic option.

\section{Darren Larsen MD}

Chief medical officer, OntarioMD, Toronto, Ont.

\section{Sarah Hutchison MHSc LLM}

Chief executive officer, OntarioMD, Toronto, Ont.

Cite as: CMAJ 2019 May 13;191:E539-40. doi: $10.1503 / \mathrm{cmaj} .71810$

Disclaimer: Until August 31, 2018, Darren Larsen was a member of the Joule Board of Directors and was not involved in the editorial decision-making process for this article.

\section{References}

1. Persaud N. A national electronic health record for primary care. CMAJ 2019;191:E28-9.

2. Collier R. National Physician Survey: EMR use at 75\%. CMAJ 2015;187: E17-18.

Competing interests: Both authors are employed by OntarioMD, which supports Ontario physicians with the adoption and use of certified electronic medical records (EMRs) and other digital health tools integrated with EMRs. OntarioMD is a wholly owned subsidiary of the Ontario Medical Association and is funded by the Ontario Ministry of Health and Long-Term Care. 